\title{
A Psicologia na Estratégia de Saúde da Família: Vivências da Residência Multiprofissional
}

Psychology in the Family Health Strategy: Experiences of the Multiprofessional Residency

La Psicología en Estrategia de Salud de la Familia: Experiencias de Residencia Multiprofesional

\section{Pâmela Kurtz Cezar, Patrícia Matte} Rodrigues \& Dorian Mônica Arpini Universidade Federal de Santa Maria

http://dx.doi.org/10.1590/1982-3703000012014 
Resumo: Este trabalho é um relato de experiência e tem por objetivo apresentar o processo de inserção da Psicologia em serviços de Estratégia de Saúde da Família numa cidade do interior do Rio Grande do Sul por meio das atividades do Programa de Residência Multiprofissional. Observou-se, durante essa vivência, que tanto os profissionais das equipes de Saúde da Família como os usuários do serviço tinham a expectativa de que a Psicologia desenvolvesse preferencialmente ações clínicas individuais. Entretanto, também foram desenvolvidas ações de maneira interdisciplinar e intersetorial, com intervenções de promoção, prevenção e educação em saúde, além de ações de articulação ensino-serviço e de articulação com serviços da rede de saúde e saúde mental do município e de participação em espaços coletivos, como Conselho Municipal de Saúde e Comissão de Saúde Mental. Conclui-se, desse modo, que, embora a Psicologia tenha ampliado suas possibilidades de atuação na área da saúde pública, ainda permanecem desafios no âmbito da atenção básica à saúde, em especial no que se refere à necessidade de superação do modelo tradicional de atuação do psicólogo, por meio de um trabalho mais compartilhado com outros profissionais da saúde.

Palavras-chave: Psicologia. Atuação do psicólogo. Saúde da Família. SUS.

Abstract: This is an experience report aiming to present the insertion process of psychology in services of the Family Health Strategy in a municipality of the countryside of Rio Grande do Sul state, Brazil, through the activities of the Multiprofessional Residency Program. During this experience, it was possible to observe that both Family Health crew professionals and users of the service expected psychology to develop individual clinical actions preferentially. However, actions of interdisciplinary and intersectoral means were also developed, with interventions of health promotion, prevention and education, besides actions of teaching-service articulation and articulation with the health and mental health network services of the municipality, as well as participation in collective spaces such as the Municipal Health Council and the Mental Health Commission. We conclude that, although psychology has expanded its performance possibilities in the area of public health, there are still challenges in the scope of primary health care, especially referring to the need to improve the traditional practicing model of the psychologist, through work shared with other health professionals.

Keywords: Psychology. Psychologist acting. Family Health. SUS.

Resumen: Este trabajo consiste en un relato de experiencia y tiene el objetivo de presentar el proceso de inserción de la psicología en servicios de Estrategia de Salud de la Familia en una ciudad del interior de Rio Grande do Sul, por medio de las actividades del Programa de Residencia Multiprofesional. Se observó, en la vivencia, que tanto los profesionales de los equipos de Salud de la Familia como los usuarios del servicio tenían la expectativa de que la psicología desarrollara preferencialmente acciones clínicas individuales. Sin embargo, también fueron desarrolladas acciones de manera interdisciplinar e intersectorial, con intervenciones de promoción, prevención y educación en salud, además de acciones de articulación enseñanza-servicio y de articulación con servicios de la red de salud y salud mental del municipio y participación en espacios colectivos, como el Consejo Municipal de Salud y la Comisión de Salud Mental. Se concluye, de tal modo, que aunque la psicología tenga ampliado sus posibilidades de actuación en el área de la salud pública, aún existen desafíos en el ámbito de la atención básica a la salud, en especial, en lo que concierne a la necesidad de superación del modelo tradicional de actuación del psicólogo, por medio de un trabajo más compartido con otros profesionales de la salud.

Palabras-clave: Psicología. Actuación del psicólogo. Salud de la Familia. SUS. 


\section{A Psicologia na saúde pública: questões históricas e atuais}

Embora a profissão de psicólogo tenha sido oficialmente regulamentada no Brasil em 1962, por meio da lei federal 4.119 , a inserção da Psicologia nos serviços de saúde é anterior a essa regulamentação, tendo seu marco de iniciação na década de 1950, por meio de práticas psicológicas pontuais na área hospitalar e da atenção materno-infantil. A partir de 1970, ampliou-se a formação em graduação e pós-graduação na área da Psicologia, e, na década de 1980, diversos concursos públicos foram abertos para psicólogos, para atuarem em serviços como hospitais, ambulatórios, unidades básicas de saúde, centros de saúde, programas de orientação, prevenção e educação em saúde (Menegon \& Coêlho, 2010; Sebastiani, 2000).

Ressalta-se que as décadas de 1970 e 1980 foram importantes não apenas para a inserção definitiva da Psicologia na saúde, como também para a história da saúde no Brasil. Nesse período, o país passou por transformações econômicas e sociais que definiram os rumos das políticas públicas de saúde. Em 1986, ocorreu a 8a Conferência Nacional de Saúde, que ampliou o conceito de saúde, passando a considerar como determinantes não só as questões biológicas mas as demandas sociais, econômicas, culturais e singulares (Brasil, 1986). Em decorrência disso, a Constituição Federal de 1988 trouxe as diretrizes para a construção do Sistema Único de Saúde (SUS) e, em 1990, duas leis federais que dispõem sobre a instituição do SUS foram sancionadas: a lei 8.080 e a lei 8.142 (Coelho, 2008).

A trajetória de construção do SUS demonstra que o conceito de saúde passou por reformulações e, ao se entender que saúde não é unicamente ausência de doença, percebeu-se que as ações em saúde precisavam ser expandidas para contemplar intervenções baseadas na integralidade do cuidado. Para tanto, foi necessário que outros profissionais se integrassem aos diversos serviços, nos três níveis de atenção à saúde: primário, secundário e terciário. Com isso, a saúde tornou-se multidisciplinar e a Psicologia passou a atuar cada vez mais no SUS (Böing \& Crepaldi, 2010; Camargo-
Borges \& Cardoso, 2005; Oliveira et al., 2004; Spink \& Matta, 2010).

Em relação à Atenção Primária à saúde, salienta-se que desde a realização da Conferência de Alma-Ata, em 1978, a Atenção Básica à saúde tem sido considerada um dos modelos prioritários para a organização de qualquer sistema de saúde (Villela et al., 2009). No Brasil, a portaria n. 648 de 2006 aprovou a Política Nacional da Atenção Básica e, em 2011, a portaria n. 2.488 estabeleceu a revisão de diretrizes e normas para a organização da Atenção Básica (AB), para a Estratégia Saúde da Família (ESF) e o Programa de Agentes Comunitários de Saúde (PACS).

A Atenção Básica é caracterizada por ações em saúde de cunho individual e coletivo que englobam intervenções de promoção, proteção, prevenção de agravos, diagnóstico, tratamento, reabilitação, redução de danos e manutenção da saúde, sendo orientadas por princípios como universalidade, acessibilidade, coordenação do cuidado, vínculo, integralidade, participação social e humanização. O trabalho é realizado em equipe e tem por alvo populações de territórios específicos, nos quais a $\mathrm{AB}$ torna-se o contato preferencial dos usuários do SUS, constituindo-se a porta de entrada e o centro de comunicação da Rede de Atenção à Saúde (Brasil, 2011).

A Política Nacional da Atenção Básica elegeu a Saúde da Família como a estratégia prioritária para a organização das ações em saúde nesse nível de atenção. Tal estratégia nasceu em 1991 com a implantação do Programa de Agentes Comunitários de Saúde. Em 1994 foi implantado o Programa de Saúde da Família, atualmente denominado Estratégia de Saúde da Família. Destaca-se que as equipes de ESF possuem composição mínima de profissionais da saúde: médico, enfermeiro, técnico e/ou auxiliar de enfermagem, agentes comunitários de saúde, podendo-se incluir cirurgião-dentista e auxiliar de saúde bucal (Brasil, 2006, 2011; Costa \& Carbone, 2004).

Identifica-se, portanto, que o psicólogo não faz parte da equipe mínima da ESF, embora a Psicologia já estivesse presente em serviços de Atenção Básica desde 1980. Somente em 2008, com a criação dos Núcleos de Apoio à 
Saúde da Família (NASF), por meio da portaria n. 154, a Psicologia passou a ser reconhecida oficialmente como profissão integrante da Atenção Básica (Jimenez, 2011). Os NASF têm a finalidade de apoiar e ampliar a abrangência, resolutividade e territorialização das ações em saúde no contexto da ESF. Assim, os diversos profissionais do NASF, dentre eles, o psicólogo, buscam a integralidade do cuidado aos usuários do SUS por meio da qualificação e complementaridade do trabalho das equipes de ESF (Brasil, 2008).

Entretanto, as práticas psicológicas desenvolvidas na Atenção Básica demonstram fragilidades no trabalho do psicólogo. Destacam-se entre essas a permanência da clínica individual, ações pouco contextualizadas e dificuldades no trabalho em equipe (Dimenstein, 1998; Jimenez, 2011; Lima, 2005; Oliveira et al., 2004).

O descompasso entre práticas psicológicas e princípios do SUS pode ser reflexo da formação acadêmica em Psicologia. As disciplinas que abordam temáticas como Saúde Coletiva e Psicologia da Saúde ainda são insuficientes e muitos psicólogos saem da graduação com uma lacuna nessas áreas. Assim, um dos desafios apresentados à Psicologia diz respeito à qualificação da formação para atuar em consonância com o SUS (Böing \& Crepaldi, 2010; Dimenstein, 1998; Dimenstein \& Macedo, 2012; Oliveira et al., 2004; Sebastiani, 2000; Spink \& Matta, 2010).

O desafio de formar profissionais mais qualificados para atuar no SUS não é exclusividade da Psicologia, mas também das demais profissões do campo da saúde (Böing \& Crepaldi, 2010; Spink \& Matta, 2010). Por conseguinte, como alternativa para superar as fragilidades na formação das profissões da área da saúde, o Ministério da Saúde, em articulação com o Ministério da Educação, criou, entre outros, o Programa de Residência Multiprofissional. A finalidade desse programa é justamente formar profissionais capazes de desenvolver intervenções ampliadas e condizentes com as diretrizes do SUS (Böing \& Crepaldi, 2010; Dimenstein \& Macedo, 2012).

Desse modo, para qualificar a atuação nos serviços de saúde pública, o psicólogo tem na residência multiprofissional uma oportunidade de formação em serviço diversificada da graduação. Para tanto, é necessário que o psicólogo consiga se reconhecer como profissional da saúde e integrante de uma equipe interdisciplinar e não somente como especialista na atenção especializada, uma vez que a transformação das práticas de cuidado em saúde está diretamente relacionada com modificações na postura profissional (Böing \& Crepaldi, 2010).

\section{Contextualização do Programa de Residência Multiprofissional}

Por meio da promulgação da lei 11.129 de 2005 foi instituída a Residência em Área Profissional de Saúde e criada a Comissão Nacional de Residência Multiprofissional em Saúde (CNRMS). As residências multiprofissionais e em área profissional da saúde são consideradas modalidades de ensino de pós-graduação lato sensu, destinadas às categorias profissionais que fazem parte da área de saúde, com exceção da Medicina. Tais categorias abrangem as seguintes profissões: Biomedicina, Ciências Biológicas, Educação Física, Enfermagem, Farmácia, Fisioterapia, Fonoaudiologia, Medicina Veterinária, Nutrição, Odontologia, Psicologia, Serviço Social e Terapia Ocupacional (Brasil, 2012).

As residências multiprofissionais são programas orientados pelos princípios e diretrizes do Sistema Único de Saúde (SUS) e consideradas um modelo de formação em serviço que busca qualificar os profissionais de saúde para atuarem de forma integral, interdisciplinar e em conformidade com as necessidades e realidades locais e regionais. Para tanto, tais programas são organizados por meio de uma carga horária de 60 horas semanais, com duração mínima de dois anos e em regime de dedicação exclusiva (Brasil, 2012).

O Programa de Residência Multiprofissional (PRM) da Universidade Federal de Santa Maria (UFSM) começou a formação de sua primeira turma em 2009, disposto em três áreas de concentração: Atenção Básica em Saúde da Família, Atenção Hospitalar e Gestão de Políticas Públicas, abrangendo os profissionais de Enfermagem, Farmácia, 
Fisioterapia, Fonoaudiologia, Nutrição, Odontologia, Psicologia, Serviço Social e Terapia Ocupacional. Atualmente também participam desse programa profissionais de Educação Física e houve modificação das áreas de concentração, que passaram a ser organizadas em três grandes eixos de atuação: PRM Integrada em Gestão e Atenção de Sistema Público de Saúde, separadas pelas ênfases de Atenção Básica/Estratégia de Saúde da Família e Vigilância em Saúde; PRM Integrada em Gestão e Atenção Hospitalar no Sistema Público de Saúde, separadas pelas ênfases de Hemato-oncologia, Materno-infantil e CrônicoDegenerativo; e, por fim, o PRM Integrada em Saúde Mental no Sistema Público de Saúde.

As intervenções dos profissionais do PRM são desenvolvidas em uma cidade de médio porte, localizada na região central do estado do Rio Grande do Sul, com população aproximada de 261 mil habitantes (IBCE, 2010). O sistema municipal de saúde, no âmbito da Atenção Básica, é composto por 18 Unidades Básicas de Saúde tradicionais e 16 equipes de ESF (totalizando 21,04\% de cobertura). Destaca-se que o município não possui NASF.

Desse modo, o PRM da UFSM prevê em sua carga horária semanal, além das atividades práticas, a participação em disciplinas, seminários, tutorias e preceptorias de campo e de núcleo. Tais atividades buscam dar suporte teórico às atividades práticas desenvolvidas pelos residentes multiprofissionais, uma vez que se trata de um programa de formação em serviço. Assim sendo, o PRM possui uma estrutura curricular que procura aproximar teoria e prática promovendo uma reflexão crítica sobre o processo de trabalho em saúde.

O currículo está organizado da seguinte maneira: os residentes do primeiro ano participam de disciplinas teóricas três vezes na semana, incluindo seminários por área de concentração. As disciplinas são realizadas no turno da noite, com duração de duas horas. As tutorias e preceptorias de campo e núcleo e as atividades práticas de formação em serviço são desenvolvidas nos campos de atuação e representam a maior parte da carga horária dos residentes $(80 \%)$. No segundo ano, os residentes participam de disciplinas teóricas uma vez na semana, além de tutorias e preceptorias de campo e núcleo, seminários preparatórios para o trabalho de conclusão do PRM e desenvolvimento do Trabalho de Conclusão de Curso, apresentado no formato de artigo de pesquisa e intervenção.

As tutorias e preceptorias são espaços de discussão e reflexão das práticas e experiências vivenciadas nos serviços, com frequência semanal e divididas em campo e núcleo de atuação. A tutoria enfatiza discussões com embasamento teórico, abrangendo temas pertinentes ao contexto em que o residente está inserido, e a preceptoria está voltada à orientação e discussão de casos, situações de saúde e/ou planejamento de atividades. Os tutores desenvolvem papel de facilitadores do processo de aprendizagem, devendo ser docentes da UFSM, com titulação mínima de Mestre. Já os preceptores são profissionais dos serviços em que os residentes atuam. No caso da Psicologia, na ênfase Atenção Básica/ Saúde da Família, a preceptoria de núcleo é desenvolvida por docentes do Curso de Psicologia, já que o município não conta com psicólogo na Atenção Básica à saúde.

O PRM/UFSM está organizado em conformidade com os princípios e diretrizes do SUS, numa perspectiva de atuação fundamentada na clínica ampliada. Inicialmente, o PRM foi constituído por meio da lógica de equipes de referência (ER) e equipes de apoio matricial (EAM). A ER é a equipe responsável pelo acompanhamento dos casos ou situações em saúde num determinado serviço e/ou território e a EAM é constituída por equipes e/ou profissionais especializados, que não fazem parte da ER, e têm a finalidade de garantir suporte a essas equipes, ampliando as possibilidades de cuidado em saúde (Campos \& Domitti, 2007).

A organização do PRM na ênfase de Atenção Básica/Saúde da Família, inicialmente, optou por distribuir os residentes multiprofissionais em três unidades de ESF do município, duas das quais eram formadas por equipes duplas. Os enfermeiros residentes ficaram fixos na ESF sendo, portanto, os profissionais de referência daquele serviço para os demais residentes, que foram considerados apoiadores matriciais. Tal distribuição se deu dessa forma por a Enfermagem ser a profissão que compõe a equipe mínima da ESF. Com isso, a Psicologia 
e demais especialidades, nesse primeiro momento, fizeram parte da equipe matricial, dando suporte às três unidades de ESF.

Posteriormente, por meio de avaliações acerca do processo de trabalho da primeira turma e com a entrada de novas turmas de residentes, a organização da ênfase de Atenção Básica/Saúde da Família sofreu modificações. A lógica da ER e da EAM permaneceu, no entanto, houve alteração e acréscimo de ESF apoiadas pelo PRM. Na terceira turma do PRM, os residentes passaram a desenvolver suas práticas em quatro ESF, localizadas em diferentes regiões do município.

Assim, as demais profissões que, na primeira turma, eram apenas de apoio matricial, passaram a compor as equipes de referência. Com isso, a Psicologia tornou-se fixa em uma das ESF, mas seguiu fazendo matriciamento a outras unidades. Destaca-se que as equipes de ESF apoiadas foram pactuadas junto à Secretaria Municipal de Saúde do município e selecionadas a partir de critérios como: disponibilidade e perfil de preceptores de campo e núcleo e perfil epidemiológico das comunidades.

Por conseguinte, este relato de experiência tem por objetivo apresentar o processo de inserção da Psicologia na Estratégia de Saúde da Família numa cidade do interior do Rio Grande do Sul, por meio das atividades do Programa de Residência Multiprofissional. Para tanto, são relatadas as trajetórias de duas psicólogas residentes, que integraram a primeira e a terceira turma do PRM na ênfase de Atenção Básica/Saúde da Família. Este relato tem por base reflexões sobre as potencialidades, fragilidades e desafios da Psicologia na saúde pública, em especial no âmbito da Atenção Básica à saúde.

\section{A experiência de inserção e as intervenções da Psicologia na Estratégia de Saúde da Família por meio do Programa de Residência Multiprofissional}

Durante 2009 a 2011, a primeira e a segunda turma de residentes multiprofissionais, na ênfase Atenção Básica/Saúde da Família, tiveram suas intervenções fragilizadas pelo fato de as equipes de ESF serem incompletas e compostas por profissionais contratados, pois apenas os Agentes Comunitários de Saúde (ACS) eram concursados e estavam entrando em exercício em 2009. Essa situação inicial tornou-se um obstáculo para os residentes pela dificuldade de articulação com as equipes, já que alguns profissionais tinham contrato por seis meses ou, no máximo, um ano.

Deste modo os princípios estruturantes da ESF como a coordenação do cuidado, a atenção longitudinal e o vínculo com as famílias e o território não eram estabelecidos no cotidiano do trabalho das unidades apoiadas pelo PRM. Somente em 2011 houve concurso público no município para profissionais de ESF e apenas a partir de 2012, com a entrada da terceira turma do PRM, o processo de trabalho entre equipe de ESF e residentes foi fortalecido.

Por sua vez, os processos de inserção dos residentes multiprofissionais nas equipes de ESF ocorreram com a necessidade de realizar um diagnóstico do território, tendo em vista que, para poder planejar e desenvolver intervenções efetivas, em especial no contexto da Atenção Primária, é necessário conhecer a comunidade, os recursos disponíveis, as demandas existentes, enfim, a realidade dos usuários, das famílias e das próprias equipes de ESF.

Tais processos são fundamentais, haja vista que a Atenção Primária à saúde tem o potencial de resolver $80 \%$ dos problemas de saúde da população, somado ao fato de ser a ordenadora das Redes de Atenção à Saúde (RAS). Salienta-se que para fazer esse diagnóstico os residentes do PRM realizaram observações, conversas formais e informais com profissionais de saúde, gestores e usuários, visitas domiciliares e reconhecimento das áreas de abrangência das equipes de ESF.

Outra atividade que fez parte desse processo foi o mapeamento da rede de serviços disponíveis no município. Os residentes se separaram por núcleo profissional e foram conhecer os dispositivos de saúde e demais setores, como Assistência Social e Educação. Em relação à Psicologia, o mapeamento compreendeu os serviços de saúde mental e outros em que havia 
psicólogo, para que fosse possível conhecer o funcionamento desses espaços e realizar articulações. Esse processo de diagnóstico e mapeamento da rede também é referido e destacado nas vivências de outros psicólogos residentes no contexto da ESF (Meira \& Silva, 2011; Soares \& Pinto, 2008).

Dentre os serviços contatados, destacam-se os Centros de Atenção Psicossocial (CAPS), incluindo-se os CAPS infantil, álcool e drogas e para adultos com neuroses graves e/ou psicoses, o Ambulatório de Saúde Mental, os Centros de Referência em Assistência Social (CRAS), os Centros de Referência Especializados de Assistência Social (CREAS) e as clínicas escola das universidades que disponibilizavam atendimento psicológico. Tal mapeamento se justifica tendo em vista a importância do trabalho em rede, preconizado pelo SUS, pois as práticas de cuidado em saúde pressupõem a capacidade de articulação com a rede de serviços (Dimenstein \& Macedo, 2012).

Após os contatos iniciais com a comunidade, as equipes de ESF e o mapeamento da rede, as intervenções da Psicologia foram discutidas e planejadas em conjunto com outros residentes, equipes de ESF e/ou em preceptoria e tutoria de campo e núcleo. Desde o princípio da atuação da Psicologia houve a preocupação de desenvolver intervenções ampliadas, para além das ações de saúde mental, contemplando as diferentes demandas e modos de vida da comunidade, considerando a influência do contexto, as potencialidades do território e das famílias que ali habitavam.

Desde o início das atividades, em especial para a Psicologia, percebeu-se certo estranhamento por parte dos profissionais das ESF e da população sobre o trabalho psicológico a ser desenvolvido. Havia expectativa de que as intervenções fossem focadas na atuação clínica individual e, inclusive, em algumas ESF, já havia lista de espera para atendimento psicoterapêutico. Nesse aspecto, visualiza-se que o entendimento que os demais profissionais de saúde e população têm acerca da Psicologia é o de que atuação psicológica é sinônimo de psicoterapia. Outras vivências de residentes psicólogos na ESF também demonstram a expectativa da equipe em relação ao atendimento clínico da Psicologia (Meira \& Silva, 2011; Soares \& Pinto, 2008).

A partir disso é possível perceber que a identidade profissional do psicólogo ainda é marcada pela atuação da clínica individual. Essa ideia estabelecida do fazer da Psicologia na saúde pode tornar os psicólogos reféns, o que, em muitos casos, faz com que mantenham um modelo de intervenção centrado na doença/ sintoma e no atendimento individual. Tal fato pode ser explicado pela maior segurança e autonomia aos psicólogos, uma vez que a formação acadêmica foi mais direcionada para esse tipo de atuação (Oliveira et al., 2004).

Assim sendo, a inserção das psicólogas residentes nas equipes ESF trouxe muitas angústias e incertezas acerca do trabalho a ser realizado, por se tratar de um novo campo de atuação para a Psicologia na cidade e pela grande demanda por atendimento psicoterapêutico vindo das equipes. Somou-se a essas dificuldades o fato de não haver psicólogo na rede básica de saúde na cidade em que o PRM desenvolve suas práticas. Os psicólogos na área da saúde estão concentrados em serviços de saúde da atenção secundária, como CAPS e Ambulatório de Saúde Mental.

Cabe salientar que essa cidade não possui NASF, o que distancia mais ainda a inserção da Psicologia nos serviços de Atenção Primária à saúde. Essa realidade que concentra os psicólogos na atenção secundária pode reforçar o modelo clínico de intervenção psicológica. Entretanto é preciso ressaltar que as ações da Psicologia em serviços de Atenção Primária ocorrem por meio da vivência de estágios na graduação ou pela formação em serviço proporcionada pelo PRM. Existem intervenções pontuais dos serviços de saúde mental, como os CAPS, na modalidade de matriciamento às equipes de ESF. Destaca-se que as experiências de matriciamento em saúde mental na Atenção Básica são práticas pioneiras e consolidadas em todo o país (Brasil, 2013; CFP, 2009; Tófoli \& Fortes, 2007).

Dentre as atividades realizadas pelas psicólogas do PRM nas equipes de ESF esteve o desenvolvimento de ações específicas da Psicologia, como atendimentos psicológicos individuais com o enfoque breve-focal e visitas 
domiciliares para avaliação e acompanhamento de usuários e famílias. A frequência dessas intervenções, avaliada conforme a demanda de cada caso, podia ser semanal, quinzenal ou mensal. Priorizavam-se as ações individuais para aquelas situações em que não havia possibilidade de acompanhamento em outro serviço ou dispositivo da rede.

A visita domiciliar (VD) foi um instrumento bastante utilizado pelas psicólogas do PRM. Por meio dela pôde-se conhecer a realidade do usuário e/ou da família de uma forma mais contextualizada. Desse modo, os casos encaminhados para a Psicologia pela equipe da ESF, em especial pelos Agentes Comunitários de Saúde, foram avaliados, num primeiro momento, por meio de VD. Os encaminhamentos eram feitos por diversos motivos: crianças que apresentavam dificuldades de aprendizagem ou hiperatividade, pessoas em isolamento social, dificuldades de relacionamento na família, violência doméstica, pessoas acamadas que estavam com dificuldades de aceitar sua atual situação, depressão, ansiedade, entre outros.

Ademais, também foram realizadas orientações a familiares e a profissionais da saúde e da educação acerca de questões de saúde mental, acolhimentos em saúde, encaminhamentos para a rede de saúde e saúde mental do município, articulação com acadêmicos de Psicologia que faziam estágio na ESF, além de apoio matricial a outras equipes de ESF que compreendiam discussões de casos e qualificação para essas equipes, por meio de um trabalho com temáticas específicas como violência, desenvolvimento infantil, apresentação da rede de saúde mental do município. Durante as vivências das duas psicólogas residentes, não foi constituído nenhum grupo específico da Psicologia nas ESF apoiadas, visto que as demandas que se apresentavam no território eram diversificadas e exigiam um cuidado ampliado e interdisciplinar.

Dentre as práticas da Psicologia com os demais profissionais do PRM e/ou com as equipes de ESF esteve o desenvolvimento de ações ampliadas, realizadas de maneira interdisciplinar e intersetorial como, por exemplo, intervenções de promoção, prevenção e educação em saúde, articulação ensinoserviço e articulação com outros dispositivos da rede disponíveis no município. Destaca-se ainda a participação em espaços coletivos, como Conselho Municipal e Local de Saúde, Conselho Municipal dos Direitos das Crianças e dos Adolescentes e Comissão de Saúde Mental. Desenvolveram-se também visitas e acompanhamentos domiciliares interdisciplinares, orientações a usuários e familiares sobre questões de saúde e garantias de direito, acolhimentos em saúde, encaminhamentos e discussões de casos entre residentes, equipe de ESF e serviços da rede como CAPS, CRAS, CREAS, Aldeias Infantis SOS, Conselho Tutelar, hospitais, escolas.

Ressalta-se a participação da Psicologia em grupos interdisciplinares, como: Grupo Hiperdia, desenvolvido para usuários com hipertensão e/ou diabetes e aberto a seus familiares; Grupo de Caminhada, oferecido para todos os usuários da comunidade; grupos para mulheres - Vida Leve e Vida Alegre -, que trabalhavam com questões de saúde na perspectiva de protagonismo das mulheres. Esses grupos eram abertos, realizados em espaços do território, com frequência semanal ou quinzenal e com a finalidade de proporcionar melhora na qualidade de vida dos participantes e seus familiares. A participação da Psicologia nesses grupos foi fundamental para trabalhar com aspectos subjetivos dos participantes e com questões de relacionamento interpessoal.

Dentre as ações ampliadas foram desenvolvidas intervenções educativas em sala de espera das ESF nas quais se discutiam questões de saúde em geral. Houve ainda a participação em campanhas de vacinação e mutirões de saúde realizados no território de abrangência das ESF, participação em eventos comemorativos nas comunidades, como dia da mulher, dia das crianças, dia mundial de combate à AIDS e apoio/fortalecimento a outras ações já desenvolvidas pelas equipes de ESF como, por exemplo, interconsultas realizadas nos atendimentos de pré-natal e puericultura. Houve ainda articulações com as escolas das comunidades para desenvolver ações pontuais em saúde para crianças, adolescentes e/ou professores.

Durante o desenvolvimento do trabalho das psicólogas residentes, percebeu-se que o profissional que atua na Atenção Básica 
precisa estar disposto a se relacionar com diferentes setores, para além da saúde, como a Assistência Social, a Educação, o Meio Ambiente, a Segurança Pública e a Justiça. Assim sendo, o profissional precisa buscar a cada nova demanda ou atividade relações de parceria para a construção de outras possibilidades de atuação, que sejam coerentes com as necessidades dos usuários e a realidade dos territórios de atuação. Contudo essa flexibilidade e abertura ao diálogo devem ser conduzidas de maneira ética e cuidadosa, respeitando princípios como direito ao sigilo e autonomia dos usuários.

Desse modo, o trabalho em equipe representa parte fundamental do trabalho dos profissionais da Atenção Básica e se apresentou como um desafio às psicólogas residentes. Realizar ações integradas e conjuntas não se configura tarefa fácil, pois exige dos profissionais características como paciência, criatividade, flexibilidade e disposição para se aventurar num espaço de construção coletiva. Bons resultados foram alcançados quando os profissionais ouviam uns aos outros, construindo, em conjunto, novas formas de cuidado.

A Atenção Básica/Saúde da Família é organizada por meio de trabalho interdisciplinar, com foco no acompanhamento de famílias num dado território (Brasil, 2011). Tal questão faz com que não se tenha a definição de um único público alvo para as intervenções. São trabalhadas políticas públicas que buscam desenvolver ações para a promoção, a prevenção, o tratamento, a reabilitação e a manutenção da saúde da população, tais como Saúde da Criança, Saúde do Adolescente, Saúde da Mulher, Saúde do Idoso, Saúde Mental, Vigilância em Saúde, Saúde na Escola, entre outras. Dessa forma, as atividades desenvolvidas pelas psicólogas residentes não se limitaram a público alvo específico, faixa etária, sexo, diagnóstico ou ações de saúde mental.

Salienta-se que ainda são poucas as publicações que descrevem a vivência de psicólogos residentes na Atenção Básica/Saúde da Família. Os artigos encontrados trazem a importância das práticas ampliadas, interdisciplinares e intersetoriais, contudo não avançam no sentido de problematizar o fazer da Psicologia para além do campo da saúde mental (Clemente et al.,
2008; Meira \& Silva, 2011; Soares \& Pinto, 2008; Tófoli \& Fortes, 2007).

Outros textos encontrados (Gama, 2009; Koda, 2009; Nepomuceno, 2009) apresentam essa reflexão, defendendo que embora as ações em saúde mental na Atenção Básica sejam fundamentais o fazer da Psicologia não pode ficar restrito a esse campo de intervenção, para que não haja uma cisão entre saúde e saúde mental. Gama (2009) reflete sobre a importância do olhar para o território, no qual o psicólogo na ESF pode ser reconhecido enquanto um psicólogo sanitarista, responsável por ações ampliadas e diversificadas.

O psicólogo residente que tem na Atenção Básica/Saúde da Família seu campo de práticas, ao final dos dois anos de residência multiprofissional torna-se um especialista em Saúde da Família e não um especialista em Saúde Mental na Atenção Básica. Portanto, a saúde mental é parte das ações do psicólogo na Atenção Básica, mas não pode ser o único cenário de atuação.

Por outro lado, é preciso destacar também que a saúde mental não pode ser um campo de prática exclusivo da Psicologia. A saúde mental precisa ser vista como um campo plural e integrado às diversas linhas de cuidado das equipes de Atenção Básica/Saúde da Família. Por esse motivo, necessita de intervenções de diversos profissionais, não apenas do psicólogo.

Na Atenção Primária à saúde trabalha-se com situações que demandam vínculo e acolhimento. A inserção da Psicologia pode contribuir para a superação do modelo de atenção centrado na doença/sintoma, pois reconhece que a saúde é perpassada pela articulação de fenômenos psicológicos, relacionais, sociais, culturais, econômicos e biológicos, possibilitando a compreensão ampliada do processo saúdedoença, numa perspectiva de integralidade do cuidado (Camargo-Borges \& Cardoso, 2005).

Além disso, a Psicologia trabalha com a escuta, ou seja, utiliza-se de tecnologias leves para compreender as singularidades dos contextos e das interações. A utilização dessas tecnologias leves é fundamental no contexto da Atenção Primária à saúde, tendo em vista que elas se caracterizam por intervenções de acolhimento, 
produção de vínculos e cuidado compartilhado, ou seja, são ações centradas na relação e na escuta. Assim, são compreendidas como tecnologias relacionais, já que acontecem no encontro entre sujeitos (Merhy, 2002).

Dessa forma, por meio das vivências das psicólogas do PRM no contexto da ESF pôdese constatar a importância e a necessidade do trabalho da Psicologia na saúde, especialmente na Estratégia de Saúde da Família, pois é onde o psicólogo pode desenvolver ações mais diversificadas que contemplem a promoção, a prevenção e a educação em saúde, demonstrando que a Psicologia pode fazer mais do que psicoterapia. Com isso, a presença do psicólogo em serviços de saúde como a ESF pode trazer e/ou fortalecer uma perspectiva ampliada de cuidado, na qual é possível integrar saúde e saúde mental, aumentando a qualidade das ações (Böing \& Crepaldi, 2010).

Desse modo, ressalta-se a importância dos PRM na formação dos profissionais da saúde. A vivência da formação em serviço possibilita a articulação de campo e núcleo de saber, qualificando o perfil dos profissionais para atuarem em equipe, de maneira corresponsável e em consonância com os princípios e diretrizes do SUS. O PRM proporciona espaços de formação interprofissional que ampliam as possibilidades de garantir cuidado integral e resolutivo. Outro ponto que merece destaque é o diferencial das vivências de residentes que têm como ênfase a Atenção Básica/Saúde da Família. Esse cenário não tem uma única linha de intervenção, portanto, possibilita uma pluralidade de ações e articulações, visto que tem no território seu contexto de atuação. O território é aqui entendido não como um espaço geográfico limitado, mas como um espaço vivo, no qual as relações se estabelecem e a vida acontece.

\section{"Velhos desafios, ainda atuais"}

Embora a experiência da Psicologia nas equipes de ESF, por meio do PRM, tenha possibilitado um olhar ampliado sobre as questões de saúde, existem desafios a serem superados, tanto no trabalho com a comunidade como no trabalho com outros profissionais de saúde. Tais desafios perpassam desde a representação que os psicólogos têm de seu trabalho, até a representação que os usuários e as equipes de saúde têm do trabalho do psicólogo. Ou seja, ainda é necessário um maior reconhecimento acerca das possibilidades de atuação da Psicologia na saúde, até mesmo para os psicólogos.

O desafio está na realização de um trabalho mais ampliado e não focado no modelo clínico individual ou nas ações exclusivas de saúde mental. Para tanto, os psicólogos precisam ter flexibilidade para trabalhar em equipe, com vistas a superar a fragmentação do conhecimento focado nas especialidades, por meio de uma relação mais aberta com as equipes de saúde.

Outro desafio encontrado pelas psicólogas do PRM foi o desenvolvimento de um trabalho mais integrado com as equipes de ESF. Em muitas situações foi difícil discutir casos e compartilhar intervenções, tendo em vista que alguns profissionais acreditavam que a Psicologia só fazia saúde mental e que as ações de saúde mental eram de responsabilidade exclusiva das psicólogas. Ademais, persiste o desafio de tornar as ações de prevenção, promoção e educação em saúde práticas cotidianas nas equipes de ESF. Essas intervenções ainda ocorrem de maneira pontual e por meio de iniciativas de alguns profissionais, residentes ou até mesmo de estagiários. Salienta-se que esses desafios são enfrentados desde as vivências da primeira turma do PRM e seguem presentes no cotidiano de atuação dos residentes de psicologia na ESF.

No que se refere aos desafios do município no contexto da Atenção Básica em saúde, destaca-se a necessidade de ampliação da cobertura de ESF, com equipes completas e profissionais concursados que trabalhem efetivamente na lógica da ESF. Nesse contexto, o processo de trabalho compreende intervenções de coordenação do cuidado, com vínculo, humanização da atenção e ampliação das ofertas em saúde. Para tanto é importante que ocorra também a implantação de Núcleos de Apoio à Saúde da Família no município.

Os NASF constituem campo de prática do PRM, sendo um dispositivo fundamental para mudança do processo de trabalho, no qual o 
psicólogo do NASF pode mediar a articulação entre psicólogo residente e equipe de ESF. Os profissionais do NASF, com o apoio dos residentes do PRM, podem contribuir no processo de educação permanente das equipes de ESF, pois a educação permanente é a ferramenta capaz de superar as práticas fragmentadas e desarticuladas ainda presentes no fazer de inúmeros profissionais e equipes de saúde.

Dentre os desafios do PRM/UFSM, está a fixação das equipes de ESF apoiadas pela Psicologia. Até então, as equipes que têm a Psicologia como referência têm mudado a cada nova turma de residentes. Essa mudança faz com que a continuidade do cuidado e o vínculo com as equipes e as comunidades sejam quebrados com o término de cada turma. Desse modo, com a chegada de novas turmas, é necessário recomeçar o processo de inserção e enfrentar as mesmas dificuldades. $\mathrm{O}$ fato de fixar algumas ESF possibilita uma melhor avaliação do trabalho realizado bem como a construção de uma história da Psicologia naquele território e nas equipes apoiadas.

\section{Considerações finais}

As vivências da Psicologia no âmbito da ESF mostraram que o trabalho vem sendo construído gradativamente desde o início do PRM e que, por se tratar de um campo de práticas novo para a Psicologia no município, muitos desafios foram encontrados nessa trajetória de inserção e atuação. Entretanto, foi possível compreender que a presença da Psicologia nesse contexto é fundamental, pois o psicólogo pode contribuir nas diversas ações de saúde, para além da saúde mental, por meio de práticas de promoção, prevenção e educação em saúde, com intervenções interdisciplinares, intersetoriais e em parceria com a comunidade.

Por outro lado, embora esse relato tenha destacado a importância de práticas ampliadas da Psicologia no contexto da ESF, não se pretendeu propor a extinção das ações de saúde mental, nem mesmo das intervenções individuais da Psicologia na Atenção Primária à saúde. O objetivo foi demonstrar as outras possibilidades de intervenção e integração, somadas à necessidade de pensar as ações de maneira contextualizada com a realidade das equipes e dos territórios de atuação.

Portanto, as experiências proporcionadas pelo PRM no contexto da ESF ajudaram a construir e a fortalecer a identidade de profissional da saúde para as psicólogas residentes, além de proporcionar um aprendizado acerca do trabalho em equipe, do compartilhamento de responsabilidades, da importância de conhecer o território antes de planejar intervenções e também da necessidade de articulação intersetorial, tendo em vista que o setor saúde não consegue sozinho dar conta das demandas que perpassam o conceito ampliado de saúde. Por conseguinte, as vivências no PRM proporcionam, não só aos psicólogos mas aos demais profissionais da residência multiprofissional uma ampliação do olhar acerca das questões de saúde e com isso foi possível visualizar que uma das alternativas para alcançar a integralidade da atenção é justamente compreender o cuidado em saúde enquanto "agir coletivo".

Por fim, destaca-se a importância do PRM como uma estratégia de mudança da postura profissional em Psicologia, tendo em vista a articulação entre prática e formação, que produz ações as quais têm impactado no processo de trabalho das equipes de ESF e, inclusive, nos cursos de graduação em Psicologia locais. Tal relação se deve ao fato de haver uma aproximação entre estagiários de Psicologia e residentes multiprofissionais nas ESF apoiadas.

Para além dessa importante articulação, salienta-se a relação com a pós-graduação, por meio do Mestrado em Psicologia, que tem como área de concentração a Psicologia da Saúde e que busca integrar temáticas de pesquisa que foram suscitadas nas experiências vivenciadas por psicólogos durante o PRM, integrando assim ensino, serviço e pesquisa. Conclui-se, portanto, que os impactos do PRM podem ser visualizados em diversos setores, não só nos cenários da saúde. 


\section{Pâmela Kurtz Cezar}

Psicóloga da Secretaria de Saúde de Santa Maria - RS. Especialista em Sistema Público de Saúde pelo Programa de Residência Multiprofissional Integrada em Sistema Público de Saúde da Universidade Federal de Santa Maria RS. Mestranda do Programa de PósGraduação em Psicologia da Universidade Federal de Santa Maria, Santa Maria - RS. Brasil. E-mail: pamelakurtz@gmail.com.

\section{Patrícia Matte Rodrigues}

Psicóloga. Especialista em Sistema Público de Saúde pelo Programa de Residência Multiprofissional Integrada em Sistema Público de Saúde da Universidade Federal de Santa Maria RS. Mestranda do Programa de Pós-Graduação em Psicologia da Universidade Federal de Santa Maria, Santa Maria - RS. Brasil. E-mail: patricia_mr@yahoo.com.br

\section{Dorian Mônica Arpini}

Psicóloga. Doutora em Psicologia Social pela Pontifícia Universidade Católica de São Paulo. Tutora da Residência Multiprofissional Integrada em Sistema Público de Saúde da Universidade Federal de Santa Maria - RS. Professora do Departamento de Psicologia e do Programa de Pós-graduação em Psicologia da Universidade Federal de Santa Maria, Santa Maria - RS. Brasil. E-mail: monica.arpini@gmail.com

\section{Endereço para envio de correspondência:}

Universidade Federal de Santa Maria. Programa de Pós-Graduação em Psicologia - Avenida Roraima no 1000, prédio 74B, sala 3208. Cidade Universitária. Bairro Camobi. CEP 97105-900 - Santa Maria, RS - Brasil. 
Referências

Böing, E., \& Crepaldi, M. A. (2010). O psicólogo na atenção básica: uma incursão pelas políticas públicas de saúde brasileiras. Psicologia: Ciência e Profissão, 30(3), 634-649.

Brasil. Ministério da Saúde. Conselho Nacional de

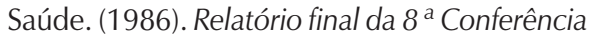
Nacional de Saúde. Brasília.

Brasil. Ministério da Saúde. (2006). Portaria no 648 de 28 de março de 2006. Aprova a Política Nacional de Atenção Básica, estabelecendo a revisão de diretrizes e normas para a organização da atenção básica para o Programa Saúde da Família (PSF) e o Programa Agentes Comunitários de Saúde (PACS). Diário Oficial da União, Brasília, DF.

Brasil. Ministério da Saúde. (2008). Portaria no 154 de 25 de janeiro de 2008. Cria os Núcleos de Apoio à Saúde da Família - NASF. Diário Oficial da União, Brasília, DF.

Brasil. Ministério da Saúde. (2011). Portaria no 2.488, de 21 de Outubro de 2011. Aprova a Política Nacional de Atenção Básica, estabelecendo a revisão de diretrizes e normas para a organização da Atenção Básica, para a Estratégia Saúde da Família (ESF) e o Programa de Agentes Comunitários de Saúde (PACS). Diário Oficial da União, Brasília, DF.

Brasil. Secretaria de Educação Superior. Comissão Nacional de Residência Multiprofissional em Saúde. (2012). Resolução no 2 de 13 de abril de 2012. Dispõe sobre Diretrizes Gerais para os Programas de Residência Multiprofissional e em Profissional de Saúde. Diário Oficial da União, Brasília, DF.

Brasil. Ministério da Saúde. Secretaria de Atenção à Saúde. Departamento de Atenção Básica. (2013). Saúde mental (Cadernos de Atenção Básica). Brasília.

Camargo-Borges, C., \& Cardoso, C. L. (2005). A psicologia e a estratégia saúde da família: compondo saberes e fazeres. Psicologia \& Sociedade, 17(2), 26-32. http://dx.doi. org/10.1590/S0102-71822005000200005.

Campos, G. W. S., \& Domitti, A. C. (2007). Apoio matricial e equipe de referência: uma metodologia para gestão do trabalho interdisciplinar em saúde. Cadernos de Saude Publica, 23(2), 399-407. http://dx.doi.org/10.1590/S0102311X2007000200016. PMid:17221089

Clemente, A., Matos, D. R., Grejanin, D. K. M., Santos, H. E., Quevedo, M. P., \& Massa, P. A.
(2008). Residência Multiprofissional em Saúde da Família e a Formação de Psicólogos para a atuação na Atenção Básica. Saúde e Sociedade, 17(1), 176-184. http://dx.doi.org/10.1590/ S0104-12902008000100016.

Coelho, I. B. (2008). Formas de pensar e organizar o Sistema de Saúde: os modelos assistenciais em saúde. In G. W. S. Campos \& A. V. P. Guerrero (Orgs.), Manual de práticas de atenção básica: saúde ampliada e compartilhada (pp. 96-131). São Paulo: Hucitec.

Conselho Federal de Psicologia - CFP. (2009). A prática da psicologia e o núcleo de apoio à saúde da família. Brasília.

Costa, E. M., \& Carbone, M. H. (2004). Saúde da Família: uma abordagem interdisciplinar. Rio de Janeiro: Rubio.

Dimenstein, M. (1998). O psicólogo nas unidades básicas de saúde: desafios para a formação e atuação profissionais. Estudos de Psicologia, 3(1), 53-82.

Dimenstein, M., \& Macedo, J. P. (2012). Formação em psicologia: requisitos para atuação na atenção primária e psicossocial [Número especial]. Psicologia: Ciência e Profissão, 32, 232-245.

Gama, C. A. P. (2009). A prática da psicologia e o núcleo de apoio à saúde da família (pp. 103110). Brasília: Conselho Federal de Psicologia.

Instituto Brasileiro de Geografia e Estatística IBGE. (2010). População de Santa Maria RS. Recuperado em 6 de agosto de 2014, de http:// www.ibge.gov.br/home/estatistica/populacao/ censo2010/tabelas_pdf/total_populacao_rio_ grande_do_sul.pdf

Jimenez, L. (2011). Psicologia na Atenção Básica à saúde: demanda território e integralidade [Número especial]. Psicologia e Sociedade, 23(spe), 129-139. http://dx.doi.org/10.1590/ S0102-71822011000400016.

Koda, M. Y. (2009). A prática da psicologia e o núcleo de apoio à saúde da família (pp. 7177). Brasília: Conselho Federal de Psicologia.

Lima, M. (2005). Atuação psicológica coletiva: uma trajetória profissional em unidade básica de saúde. Psicologia em Estudo, 10(3), 431-440. http://dx.doi.org/10.1590/S141373722005000300011.

Meira, M. A., \& Silva, M. O. (2011). Atuação da psicologia na estratégia saúde da família: 
a Experiência de um psicólogo em uma residência multiprofissional. Revista Brasileira de Ciências da Saúde, 15(3), 369-376. http:// dx.doi.org/10.4034/RBCS.2011.15.03.12.

Menegon, V. S. M., \& Coêlho, A. E. L. (2010). Psicologia e sua inserção no sistema público de saúde: um painel longitudinal de temasfoco publicados em periódicos brasileiros. In M. J. P. Spink (Org.), A psicologia em diálogo com o SUS: prática profissional e produção acadêmica (pp. 175-205). São Paulo: Casa do Psicólogo.

Merhy, E. E. (2002). Saúde: a cartografia do trabalho vivo. São Paulo: Hucitec.

Nepomuceno, L. B. (2009). A prática da psicologia e o núcleo de apoio à saúde da família (pp. 4959). Brasília: Conselho Federal de Psicologia.

Oliveira, I. F., Dantas, C. M., Costa, A. L. F., Alvarenga, A. R., Carvalho, D. B., \& Yamamoto, O. H. (2004). O psicólogo nas unidades básicas de saúde: formação acadêmica e prática profissional. Interações, 9(17), 71-89.

Sebastiani, R. W. (2000). Histórico e evolução da psicologia numa perspectiva Latino Americana.
In V. A. Angerami-Camon (Ed.), Psicologia da saúde (pp. 201-222). São Paulo: Pioneira.

Soares, N. M., \& Pinto, M. E. B. (2008). Interfaces da psicologia aplicada à saúde: atuação da psicologia na estratégia saúde da família em Londrina. Revista da Sociedade Brasileira de Psicologia Hospitalar, 11(2), 89-100.

Spink, M. J., \& Matta, G. C. (2010). A prática profissional Psi na saúde pública: configurações históricas e desafios contemporâneos. In M. J. P. Spink (Org.), A psicologia em diálogo com o SUS: prática profissional e produção acadêmica (pp. 25-51). São Paulo: Casa do Psicólogo.

Tófoli, L. F. \& Fortes, S. (2007). Apoio matricial de saúde mental na atenção primária no município de Sobral, CE: o relato de uma experiência. SANARE: Revista de Políticas Públicas, 6(2), 34-42.

Villela, W. V., Araújo, E. C., Ribeiro, S. A., Cuginotti, A. P., Hayana, E. T., Brito, F. C., et al (2009). Desafios da atenção básica em saúde: a experiência de Vila Mariana, São Paulo, Brasil. Cadernos de Saude Publica, 25(6), 1316-1324. http://dx.doi. org/10.1590/S0102-311X2009000600014. PMid:19503962 